\title{
Terahertz induced optical second harmonic generation from dielectric interfaces: mechanism and application
}

\author{
S.B. Bodrov $^{1,2}$ Yu.A. Sergeev ${ }^{2}$, A.I. Korytin ${ }^{2}$, M.Y. Emelin ${ }^{2}$, M.Y. Ryabikin ${ }^{2}$ \\ and A.N. Stepanov ${ }^{2}$

\begin{abstract}
${ }^{1}$ University of Nizhny Novgorod, Nizhny Novgorod 603950 Russia, bosbor@ufp.appl.sci-nnov.ru
\end{abstract} \\ ${ }^{2}$ Institute of Applied Physics of the Russian Academy of Sciences, Nizhny Novgorod 603950 Russia
}

Optical second harmonic ( $\mathrm{SH})$ generation from interfaces of different materials is widely used for surface studies. The surface breaks the symmetry allowing SH generation even in isotropic media. The quality of the surface as well as presence of an additional layers on the surface significantly influence on $\mathrm{SH}$ generation. Also, it was shown that application of constant external electric field changes the magnitude of the generated SH signal [1]. In Ref. [2] it was proposed to use a weak $\mathrm{THz}$ electric field to modulate $\mathrm{SH}$ generation from surface of nontransparent optical material (silicon) as a method to measure $\mathrm{THz}$ field waveform. Here we propose to use $\mathrm{THz}$ induced $\mathrm{SH}$ generated by femtosecond laser radiation to detect optically hidden layers (glue) placed not only on the surface but also into a bulk of transparent materials (quartz). We showed that existence of terahertz radiation influences on SH generation only from the interfaces (glue-quartz, air-quartz) and not from the bulk of the quartz. To explain this effect a model based on interaction of electromagnetic radiation with an electron in coulomb potential biased by embedded surface electric field was proposed and analyzed.

A Ti:Sapphire laser system ( $1 \mathrm{~mJ}, 1 \mathrm{kHz}, 70 \mathrm{fs})$ was used in our experiment. The optical beam was divided into two parts (see Fig. 1). The first pulse generated $\mathrm{THz}$ radiation in a $\mathrm{LiNbO} 3$ crystal by the tilted-front technique [3]. The $\mathrm{THz}$ pulse (the waveform is shown in Fig. 2(a)) was focused on the sample collinear with a probe (weak) femtosecond optical pulse. The sample was fabricated by cementing of two $0.9 \mathrm{~mm}$ fused quartz plates by glue with thickness of about several $\mu \mathrm{m}$. The generated optical $\mathrm{SH}$ was detected by PMT in reflection and transmittance setups. In the first setup the sample was placed at $45^{\circ}$ to incident probe pulse and PMT detected reflected optical $\mathrm{SH}$. In the second setup the probe optical pulse propagated normally to the incident surface of the sample and PMT detected transmitted optical SH.

Figure 2(b) shows the $\mathrm{SH}$ signal as a function of delay time between optical and $\mathrm{THz}$ pulses in the transmittance setup (the similar signal but two orders less magnitude was measured in the reflection setup). Due to different velocities of $\mathrm{THz}$ and optical pulses, their interaction occurs at different points inside the sample. The measured SH signal at $t=0$ corresponds to the signal from the entrance surface of the sample, at $t=1.4 \mathrm{ps}$ - from the interface glue-quartz, and at $t=2.7 \mathrm{ps}-$ from the output boundary of the sample. The dependence obtained in Fig. 2 (b) demonstrates the possibility of determining the presence of internal boundaries in the material that are not visible in the optical range. With the known values of the refractive indexes for optical and $\mathrm{THz}$ radiation, one can find a depth of internal inhomogeneity.

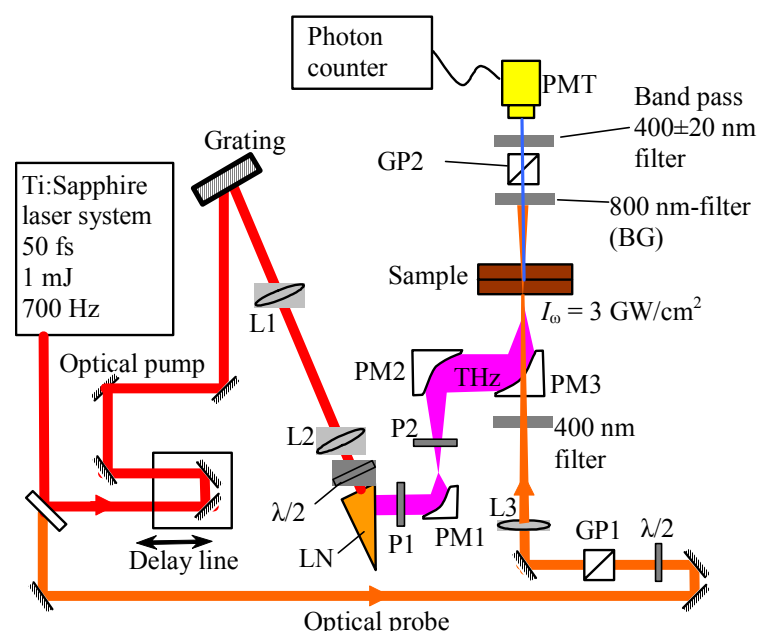

Fig. 1. (a) Experimental setup. $\mathrm{LN}-\mathrm{LiNbO}_{3}$ crystal; PM1, PM2 and PM3 - parabolic mirrors; GP1,GP2 - Glan prisms; PMT - photo multiplier tube; P1, P2 - THz polarizers.
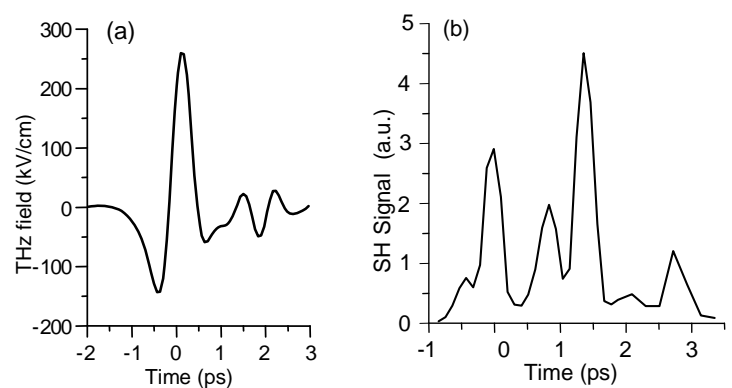

Fig. 2. (a) $\mathrm{THz}$ waveform. (b) $\mathrm{THz}$ induced optical $\mathrm{SH}$ generation from cemented quarts plates.

The fundamental feature of the dependence in Fig. 2(b) is absence (or negligible small value) of $\mathrm{SH}$ signal from the bulk. Indeed, the $\mathrm{SH}$ in the bulk of isotropic material can be generated by the fundamental harmonic via cubic nonlinearity in the presents of external constant (or low-frequency) field. Thus, the optical and THz pulses should intersect in space. Due to material dispersion the optical and $\mathrm{THz}$ pulses interact in the bulk on a walk-off distance $L_{w}=c \tau /\left(n_{T H z}-n_{\text {opt }}\right)$, where $\tau-\mathrm{THz}$ pulse duration, $n_{T H z}$ and $n_{\text {opt }}$ - terahertz and optical refraction coefficients, respectively. Taking $\tau=500$ fs we have for fused quartz $L_{w}=300 \mu \mathrm{m}$. Changing the time delay results to moving the position of interaction region inside the sample. If the thickness of the sample (1.8 
$\mathrm{mm}$ ) is more than the walk-off length (as in our experiment) the dependence of SH signal on time delay should have step-like profile with rising and falling times $\tau \approx 0.5 \mathrm{ps}$ (with possible modulation due to short $\sim 10 \mu \mathrm{m}$ coherent length between first and second optical harmonics). Such signal was not measured in the experiment. At the same time, the signal tracing the form of $\mathrm{THz}$ intensity was well observed that indicates the presents of a source of the second harmonic only near the interfaces.

To qualitatively explain the above-discussed experimental result we considered a nonlinear response of hydrogen-like atom in the field of optical and $\mathrm{THz}$ radiation. The presence of the interface was modeled by applying additional constant field with corresponding orientation. The simulation was based on 2D and 3D numerical solution of Schrödinger equation. It was shown that near the surface the SH response has a resonance defined by the value of the constant field. Switching-off of the constant field (that corresponds to moving atom into the bulk) leads to a decrease in the second harmonic by several orders of magnitude in agreement with experimental data. This singleparticle theory gives a good qualitative explanation of experimental results. For more accuracy (and better quantitative agreement with experimental data) one should take into account the number of atoms in the bulk and near the surface as well as the dynamics of their radiation.

This work was supported in part by the RFBR, research project No. 18-02-00968.

\section{References}

[1] O. A. Aktsipetrov, A. A. Fedyanin, A. V. Melnikov, E. D. Mishina, A. N. Rubtsov, M. H. Anderson, P. T. Wilson, M. ter Beek, X. F. Hu, J. I. Dadap, and M. C. Downer. Dc-electric-field-induced and low-frequency electromodulation second-harmonic generation spectroscopy of $\mathrm{Si}(001)-\mathrm{SiO} 2$ interfaces // Phys. Rev. B. 1999. V. 60. P. 8924.

[2] A. Nahata and T.F. Heinz. Detection of freely propagating terahertz radiation by use of optical secondharmonic generation. // Opt. Lett. 1998. V. 23. P. 67.

[3] S.B. Bodrov, A.A. Murzanev, Y.A. Sergeev, Y.A. Malkov, and A.N. Stepanov. Terahertz generation by tiltedfront laser pulses in weakly and strongly nonlinear regimes. // Appl. Phys. Lett. 2013. V. 103. P. 251103. 